\title{
Dynamics of a massive intruder in a homogeneously driven granular fluid
}

\author{
A. Puglisi - A. Sarracino - G. \\ Gradenigo · D. Villamaina
}

Received: date / Accepted: date

\begin{abstract}
A massive intruder in a homogeneously driven granular fluid, in dilute configurations, performs a memory-less Brownian motion with drag and temperature simply related to the average density and temperature of the fluid. At volume fraction $\sim 10-50 \%$ the intruder's velocity correlates with the local fluid velocity field: such situation is approximately described by a system of coupled linear Langevin equations equivalent to a generalized Brownian motion with memory. Here one may verify the breakdown of the FluctuationDissipation relation and the presence of a net entropy flux - from the fluid to the intruder - whose fluctuations satisfy the Fluctuation Relation.
\end{abstract}

Keywords Granular materials · Non-equilibrium fluctuations

Granular fluids represent a valid benchmark for modern theories of nonequilibrium statistical mechanics [9]. Due to dissipative interactions among the microscopic constituents, energy is not conserved and an external source is necessary to maintain a stationary state. The consequence is a breakdown of time reversal invariance and the failure of properties such as the Equilibrium Fluctuation-Dissipation relation (EFDR) 6]. In recent years, a systematic theory for the dilute limit has been developed, in good agreement with numerical simulations 2, 1, while a general understanding of dense granular fluids is still lacking. A common approach is the so-called Enskog correction [2,3, which reduces the breakdown of Molecular Chaos to a renormalization of the collision frequency. In cooling regimes, the Enskog theory may describe strong

\footnotetext{
All authors

CNR-ISC c/o Dip. di Fisica,

Universita' degli Studi di Roma "La Sapienza",

Piazzale Aldo Moro 2, I-00185 Roma, Italy.

Tel.:+39-0649913508 Fax:+39-064463158

E-mail: andrea.puglisi@roma1.infn.it

E-mail: ale.sarracino@gmail.com

E-mail: ggradenigo@gmail.com

E-mail: villamaina@gmail.com
} 
non-equilibrium effects, due to the explicit cooling time-dependence 22]. Nevertheless it cannot describe dynamical effects in stationary regimes, such as multiple characteristic times or different decays of response and autocorrelation [5, 18 .

Here we review a recent model [24] for the dynamics of a massive tracer moving in a gas of smaller granular particles, both coupled to an external bath. Taking as reference point the dilute limit, where the system has a closed analytical description [23], a Langevin equation linearly coupled to a fluctuating local velocity field is proposed as first approximation capable of describing the dense case. Its main features are: i) the decay of correlation and response functions is not simply exponential and shows backscattering [15, 4 and ii) the EFDR [10,13, of the first and second kind do not hold. In such a model, detailed balance is not necessarily satisfied, and a fluctuating entropy production [25] can be measured, which fairly verifies the Fluctuation Relation [11, 12,13 .

The model reviewed here is the following: an "intruder" disc of mass $m_{0}=$ $M$ and radius $R$, moving in a gas of $N$ granular discs with mass $m_{i}=m$ $(i>0)$ and radius $r$, in a two dimensional box of area $A=L^{2}$. We denote by $n=N / A$ the number density of the gas and by $\phi$ the occupied volume fraction, i.e. $\phi=\pi\left(N r^{2}+R^{2}\right) / A$ and we denote by $\boldsymbol{V}$ (or $\boldsymbol{v}_{0}$ ) and $\boldsymbol{v}$ (or $\boldsymbol{v}_{i}$ with $i>0$ ) the velocity vector of the tracer and of the gas particles, respectively. Interactions among the particles are hard-core binary instantaneous inelastic collisions, such that particle $i$, after a collision with particle $j$, comes out with a velocity $\boldsymbol{v}_{i}^{\prime}=\boldsymbol{v}_{i}-(1+\alpha) \frac{m_{j}}{m_{i}+m_{j}}\left[\left(\boldsymbol{v}_{i}-\boldsymbol{v}_{j}\right) \cdot \hat{\boldsymbol{n}}\right] \hat{\boldsymbol{n}}$ where $\hat{\boldsymbol{n}}$ is the unit vector joining the particles' centers of mass and $\alpha \in[0,1]$ is the restitution coefficient $(\alpha=1$ is the elastic case). The mean free path of the intruder is proportional to $l_{0}=1 /(n(r+R))$ and we denote by $\tau_{c}$ its mean collision time. Two kinetic temperatures can be introduced for the two species: the gas temperature $T_{g}=m\left\langle\boldsymbol{v}^{2}\right\rangle / 2$ and the tracer temperature $T_{t r}=M\left\langle\boldsymbol{V}^{2}\right\rangle / 2$.

The equation of motion of the $i$-th particle reads: $m_{i} \dot{v}_{i}(t)=-\gamma_{b} v_{i}(t)+$ $f_{i}(t)+\xi_{b}(t)$. Here $f_{i}(t)$ is the force taking into account the collisions of particle $i$ with other particles, and $\xi_{b}(t)$ is a white noise (different for all particles), with $\left\langle\xi_{b}(t)\right\rangle=0$ and $\left\langle\xi_{b}(t) \xi_{b}\left(t^{\prime}\right)\right\rangle=2 T_{b} \gamma_{b} \delta\left(t-t^{\prime}\right)$. The effect of the external energy source balances the energy lost in the collisions and a stationary state is attained with $m_{i}\left\langle v_{i}^{2}\right\rangle \leq T_{b}$ [27,14, 19, 16, 8 .

At low packing fractions, $\phi<0.1$, and in the large mass limit, $m / M \ll 1$, using the Enskog approximation it has been shown [23] that the dynamics of the intruder is described by a linear Langevin equation:

$$
M \dot{V}=-\Gamma_{E} V+\mathcal{E}_{E},
$$

with $\mathcal{E}_{E}$ a white noise with $\left\langle\mathcal{E}_{E}\right\rangle=0,\left\langle\mathcal{E}_{E}(t) \mathcal{E}_{E}\left(t^{\prime}\right)\right\rangle=2 \delta\left(t-t^{\prime}\right) \Gamma_{E} T_{t r}^{E}$ and $T_{t r}^{E}=$ $\left(\gamma_{b} T_{b}+\gamma_{g}^{E} \frac{1+\alpha}{2} T_{g}\right) / \Gamma_{E}$ is the tracer's temperature. In this limit the velocity autocorrelation function shows a simple exponential decay, with characteristic time $M / \Gamma_{E}$, where $\Gamma_{E}=\gamma_{b}+\gamma_{g}^{E}$ and $\gamma_{g}^{E}=\frac{g_{2}(r+R)}{l_{0}} \sqrt{2 \pi m T_{g}}(1+\alpha)$ where $g_{2}(r+R)$ is the pair correlation function for a gas particle and the intruder 
at contact. Time-reversal and the EFDR, weakly modified for uniform dilute granular gases [17,5,21, become perfectly satisfied for a massive intruder.

As the packing fraction is increased, the Enskog approximation fails in predicting dynamical properties. In particular, velocity autocorrelation $C(t)=$ $\langle V(t) V(0)\rangle /\left\langle V^{2}\right\rangle$ and linear response function $R(t)=\overline{\delta V(t)} / \delta V(0)$ show an exponential decay modulated by oscillating functions 4, 24. Moreover violations of the EFDR $C(t)=R(t)$ are observed for $\alpha<1$ [18,26]. The Enskog approximation is unable to explain the observed functional forms, because it only modifies by a constant factor the collision frequency [2,23]: a model with more than one characteristic time is needed. A first approximation is given by an auxiliary field coupled to the intruder's velocity:

$$
\begin{aligned}
M \dot{V} & =-\Gamma_{E}(V-U)+\sqrt{2 \Gamma_{E} T_{g}} \mathcal{E}_{V} \\
M^{\prime} \dot{U} & =-\Gamma^{\prime} U-\Gamma_{E} V+\sqrt{2 \Gamma^{\prime} T_{b}} \mathcal{E}_{U}
\end{aligned}
$$

where $\mathcal{E}_{V}$ and $\mathcal{E}_{U}$ are white noises of unitary variance. Two new parameters appear: the mass of the local field $M^{\prime}$ and its drag coefficient $\Gamma^{\prime}$. The dilute limit here is obtained for $\Gamma^{\prime} \sim M^{\prime} \rightarrow \infty$. In such a limit indeed $U \rightarrow 0$ and the equation for $V$ comes back in the form discussed above 23. In such a form (2), the dynamics of the tracer is remarkably simple: indeed $V$ follows a Langevin equation in a Lagrangian frame with respect to a field $U$, which is the local average velocity field of the gas particles colliding with the tracer. A first justification of this model comes from realizing [24] that it is equivalent to a Generalized Langevin Equation with exponential memory, which is consistent with a typical approximation done for Brownian Motion when, at high densities, the coupling of the intruder with fluid hydrodynamic modes, decaying exponentially in time (see [28, Cap. 8.6 and 9.1), must be taken into account. Such a coupling, which in principle involves a continuum of modes, is reduced here to a single dominant mode: this is sufficient to introduce a new non-trivial timescale. The full coupling would reproduce finer features which become relevant at larger densities or larger times, such as long-time powerlaw tails. The fact that the "temperature" of the local velocity field $U$ is equal to the bath temperature $T_{b}$ comes as a consequence of the conservation of momentum in collisions, implying that the average velocity of a group of particles is not changed by collisions among themselves and is only affected by the external bath and a (small) number of collisions with outside particles. This scenario is fully consistent with recent study of hydrodynamic fluctuations for the velocity field of the same fluid model [7,8].

A stronger justification comes, however, from its effectivness in reproducing the numerical results, as detailed in 24]. From the simulations it is seen that the relaxation time of the local field $\tau_{U}=M^{\prime} / \Gamma^{\prime}$, rescaled by the mean collision time, increases with the packing fraction and with the inelasticity, as expected. At high densities it appears that $\Gamma^{\prime} \sim 1 / \phi$, and $T_{t r} \sim T_{g} \sim T_{g}^{E}$, likely due to stronger correlations among particles. At large $\phi$ we observe $T_{t r}>T_{t r}^{E}$, consistent with a smaller dissipation for correlated collisions. Model (2) predicts 
$C=f_{C}(t)$ and $R=f_{R}(t)$ with

$$
f_{C(R)}=e^{-g t}\left[\cos (\omega t)+a_{C(R)} \sin (\omega t)\right] .
$$

The variables $g, \omega, a_{C}$ and $a_{R}$ are known algebraic functions of $\Gamma_{E}, T_{g}, \Gamma^{\prime}, M^{\prime}$ and $T_{b}$. In particular, the ratio $a_{C} / a_{R}=\left[T_{g}-\Omega\left(T_{b}-T_{g}\right)\right] /\left[T_{g}+\Omega\left(T_{b}-T_{g}\right)\right]$, with $\Omega=\Gamma_{E} /\left(\left(\Gamma^{\prime}+\Gamma_{E}\right)\left(\Gamma_{E} M^{\prime} / M-\Gamma^{\prime}\right)\right)$. Hence, in the elastic $\left(T_{g} \rightarrow T_{b}\right)$ as well as in the dilute limit $\left(\Gamma^{\prime} \rightarrow \infty\right)$, one gets $a_{C}=a_{R}$ and recovers the EFDR $C(t)=R(t)$. Such predictions are all verified in numerical simulations 24. In particular Fig. 1 depicts correlation and response functions in a dense case (elastic and inelastic): symbols correspond to numerical data and continuous lines the analytical curves. In the inelastic case, deviations from $\operatorname{EFDR} R(t)=$ $C(t)$ are observed. In the inset of Fig. 1 the ratio $R(t) / C(t)$ is also reported. It is important to notice that the main responsibility for the breakdown of the EFDR is the coupling between $V$ and $U$, indeed Eq. (3) can be expressed in a different way: $R(t)=a C(t)+b\langle V(t) U(0)\rangle$ with $a=\left[1-\left(T_{g}-T_{b}\right) \Omega_{a} / \Gamma^{\prime}\right]$ and $b=\left(T_{g}-T_{b}\right) \Omega_{b}$, where $\Omega_{a}$ and $\Omega_{b}$ are known functions of the parameters. At equilibrium or in the dilute limit the EFDR is recovered.
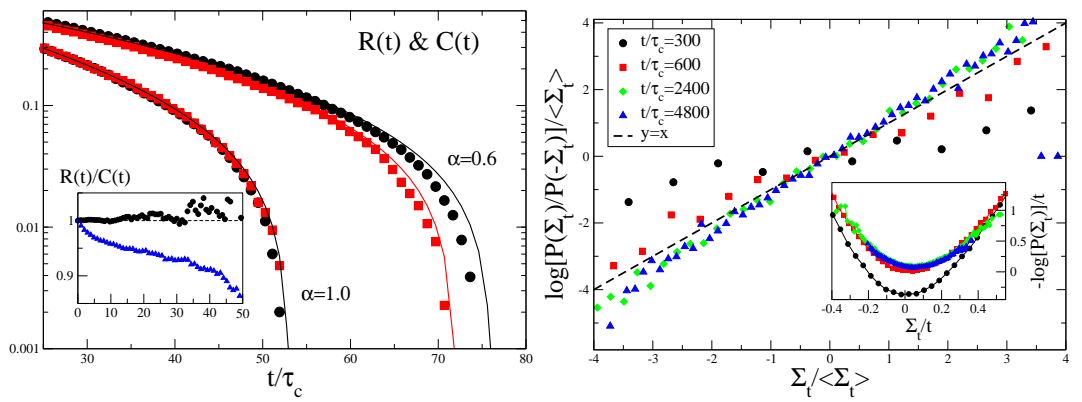

Fig. 1 (Color online). Left: correlation function $C(t)$ (black circles) and response function $R(t)$ (red squares) for $\alpha=1$ and $\alpha=0.6$, at $\phi=0.33$. Continuous lines show curves obtained with Eqs. (3). Inset: the ratio $R(t) / C(t)$ is reported in the same cases. Right: Check of the fluctuation relation (5) in the system with $\alpha=0.6$ and $\phi=0.33$. Inset: collapse of the rescaled probability distributions of $\Sigma_{t}$ at large times onto the large deviation function.

An important independent assessment of model (2) comes from the study of the fluctuating entropy production 25] which quantifies the deviation from detailed balance in a trajectory. Given the trajectory in the time interval $[0, t],\{V(s)\}_{0}^{t}$, and its time-reversed $\{\mathcal{I} V(s)\}_{0}^{t} \equiv\{-V(t-s)\}_{0}^{t}$, the entropy production for our model takes the form 20.

$$
\Sigma_{t}=\log \frac{P\left(\{V(s)\}_{0}^{t}\right)}{P\left(\{\mathcal{I} V(s)\}_{0}^{t}\right)} \approx \Gamma_{E}\left(\frac{1}{T_{g}}-\frac{1}{T_{b}}\right) \int_{0}^{t} d s V(s) U(s) .
$$

This functional vanishes exactly in the elastic case, $\alpha=1$, where equipartition holds, $T_{g}=T_{b}$, and is zero on average in the dilute limit, where $\langle V U\rangle=0$. 
Formula (44) reveals that the leading source of entropy production is the energy transferred by the "force" $\Gamma_{E} U$ on the tracer, weighed by the difference between the inverse temperatures of the two "thermostats". Therefore, to measure entropy production, we need to measure the fluctuations of $U$ : a possible choice is a local average of particles' velocities in a circle of radius $l+R$ centered on the tracer. Details on how to choose in a reliable way the correct $l$ are given in 24]. Following such procedure, in the case $\phi=0.33$ and $\alpha=0.6$, we estimate for the correlation length $l \sim 9 r \sim 6 l_{0}$. Then, measuring the entropy production from Eq. (4) along many trajectories of length $t$, we computed the probability $P\left(\Sigma_{t}=x\right)$ and compared it to $P\left(\Sigma_{t}=-x\right)$, in order to verify the Fluctuation Relation [11,12,13.

$$
\log \frac{P\left(\Sigma_{t}=x\right)}{P\left(\Sigma_{t}=-x\right)}=x .
$$

In the right frame of Fig. 1 the results of this comparison are reported. The main frame confirms that at large times the Fluctuation Relation (5) is well verified within the statistical errors. The inset shows the collapse of $\log P\left(\Sigma_{t}\right) / t$ onto the large deviation rate function for large times. Notice that - in formula (4) - a wrong evaluation of the weighing factor $\left(1 / T_{g}-1 / T_{b}\right)$ or of the "energy injection rate" $\Gamma_{E} U(t) V(t)$ in Eq. (4) could produce a completely different slope in Fig. 11 (right frame).

To conclude this paper, we stress that velocity correlations $\left\langle V(t) U\left(t^{\prime}\right)\right\rangle$ between the intruder and the surrounding velocity field are responsible for both the violations of the EFDR and the appearance of a non-zero entropy production, provided that the two fields are at different temperatures. We also mention that larger violations of EFDR can be observed using an intruder with a mass equal or similar to that of other particles [18, with the important difference that in such a case a simple "Langevin-like" model for the intruder's dynamics is not available.

Acknowledgements This work is dedicated to the memory of Isaac Goldhirsch, from whom we learned plenty of science, but also a smiling attitude toward serious things. The work is supported by the "Granular-Chaos" project, MIUR grant number RBID08Z9JE.

\section{References}

1. Brey, J.J., Maynar, P., de Soria, M.I.G.: Fluctuating hydrodynamics for dilute granular gases. Phys. Rev. E 79, 051,305 (2009)

2. Brilliantov, N.K., Poschel, T.: Kinetic Theory of Granular Gases. Oxford University Press (2004)

3. Dufty, J.W., Santos, A.: Dynamics of a hard sphere granular impurity. Phys. Rev. Lett. 97, 058,001 (2006)

4. Fiege, A., Aspelmeier, T., Zippelius, A.: Long-time tails and cage effect in driven granular fluids. Phys. Rev. Lett. 102, 098,001 (2009)

5. Garzó, V.: On the Einstein relation in a heated granular gas. Physica A 343, 105 (2004)

6. Goldhirsch, I., van Noije, T.P.C.: Green-kubo relations for granular fluids. Phys. Rev. E 61, 3241 (2000) 
7. Gradenigo, G., Sarracino, A., Villamaina, D., Puglisi, A.: Fluctuating hydrodynamics and correlation lengths in a driven granular fluid. J. Stat. Mech. p. P08017 (2011)

8. Gradenigo, G., Sarracino, A., Villamaina, D., Puglisi, A.: Growing non-equilibrium length in granular fluids: from experiment to fluctuating hydrodynamics. Europhys.Lett. 96, 14,004 (2011)

9. Jaeger, H.M., Nagel, S.R., Behringer, R.P.: Granular solids, liquids, and gases. Rev. Mod. Phys. 68, 1259 (1996)

10. Kubo, R., Toda, M., Hashitsume, N.: Statistical physics II: Nonequilibrium stastical mechanics. Springer (1991)

11. Kurchan, J.: Fluctuation theorem for stochastic dynamics. J. Phys. A 31, 3719 (1998)

12. Lebowitz, J.L., Spohn, H.: A Gallavotti-Cohen-type symmetry in the large deviation functional for stochastic dynamics. J. Stat. Phys. 95, 333 (1999)

13. Marconi, U.M.B., Puglisi, A., Rondoni, L., Vulpiani, A.: Fluctuation-dissipation: Response theory in statistical physics. Phys. Rep. 461, 111 (2008)

14. van Noije, T.P.C., Ernst, M.H., Trizac, E., Pagonabarraga, I.: Randomly driven granular fluids: Large-scale structure. Phys. Rev. E 59, 4326 (1999)

15. Orpe, A.V., Kudrolli, A.: Phys. Rev. Lett. 98, 238,001 (2007)

16. Prevost, A., Egolf, D.A., Urbach, J.S.: Forcing and velocity correlations in a vibrated granular monolayer. Phys. Rev. Lett. 89, 084,301 (2002)

17. Puglisi, A., Baldassarri, A., Loreto, V.: Fluctuation-dissipation relations in driven granular gases. Physical Review E 66, 061305 (2002)

18. Puglisi, A., Baldassarri, A., Vulpiani, A.: Violations of the Einstein relation in granular fluids: the role of correlations. J. Stat. Mech. p. P08016 (2007)

19. Puglisi, A., Loreto, V., Marconi, U.M.B., Petri, A., Vulpiani, A.: Clustering and nongaussian behavior in granular matter. Phys. Rev. Lett. 81, 3848 (1998)

20. Puglisi, A., Villamaina, D.: Irreversible effects of memory. Europhys. Lett. 88, 30,004 (2009)

21. Puglisi, A., Visco, P., Trizac, E., van Wijland, F.: Dynamics of a tracer granular particle as a nonequilibrium markov process. Phys. Rev. E 73, 021,301 (2006)

22. Santos, A., Dufty, J.W.: Critical behavior of a heavy particle in a granular fluid. Phys. Rev. Lett. 86, 4823 (2001)

23. Sarracino, A., Villamaina, D., Costantini, G., Puglisi, A.: Granular brownian motion. J. Stat. Mech. p. P04013 (2010)

24. Sarracino, A., Villamaina, D., Gradenigo, G., Puglisi, A.: Irreversible dynamics of a massive intruder in dense granular fluids. Europhys. Lett. 92, 34,001 (2010)

25. Seifert, U.: Entropy production along a stochastic trajectory and an integral fluctuation theorem. Phys. Rev. Lett. 95, 040,602 (2005)

26. Villamaina, D., Puglisi, A., Vulpiani, A.: The fluctuation-dissipation relation in subdiffusive systems: the case of granular single-file diffusion. J. Stat. Mech. p. L10001 (2008)

27. Williams, D.R.M., MacKintosh, F.C.: Driven granular media in one dimension: Correlations and equation of state. Phys. Rev. E 54, R9 (1996)

28. Zwangzig, R.: Nonequilibrium statistical mechanics. Oxford Univ. Press (2001) 